\title{
Assessing stereomotion thresholds with a high-resolution computer monitor
}

\author{
P. SERVOS, L. A. SYMONS, W. SCHMIDT, and M. A. GOODALE \\ University of Western Ontario, London, Ontario, Canada
}

\begin{abstract}
We investigated the feasibility of a computer-graphics-based method of assessing stereomotion thresholds (Silicon Graphics Stereoview stereoscopic system). Stereomotion thresholds for a rectangle oscillating in depth were determined with the use of a dual randomly interleaved staircase design. In a group of 31 naive observers, the average thresholds of $5.97^{\circ}$ of arc for crossed stereomotion and $6.00^{\prime}$ of arc for uncrossed stereomotion were comparable to those assessed in earlier work done with optics-based techniques. By assessing the thresholds for a rectangle that was defined either by lateral motion or by changing size, in a group of experienced observers, we were able to show that any potential residual translational motion present in the display would not have influenced the stereomotion thresholds. Our findings suggest that this computer-graphics-based technique may be a reasonable alternative to optics-based methods of assessing stereomotion thresholds.
\end{abstract}

Work by Richards and Regan in the early 1970 s demonstrated that humans not only are sensitive to visual stimuli defined by static stereopsis (i.e., at fixed disparities) but also are able to process stimuli on the basis of changing disparity (Richards, 1972; Richards \& Regan, 1973). This phenomenon has been termed stereomotion (see Regan, Frisby, Poggio, Schor, \& Tyler, 1990). Stereomotion is based on the principle that as an object moves toward (or away from) an observer, it produces retinal images that are moving in opposite directions in the two eyes. The difference in retinal image velocities in the left and right eyes (and hence changing disparity) can produce a compelling sensation of motion in depth. Indeed, when each eye is presented with separate images moving in opposite directions, a compelling sensation of motion in depth can be produced even though the "object" is not physically moving in 3-D space or changing in size (Beverley \& Regan, 1973, 1975; Regan \& Beverley, 1979; Regan, Beverley, \& Cynader, 1979; Richards, 1972; Richards \& Regan, 1973).

One important contribution of stereomotion might occur in the processing of information about direction in depth (see Regan, 1991, 1992; Regan et al., 1990). This sort of information is important for the control of skilled visuomotor behaviors, such as in landing aircraft or in the precise interceptive movements in sports such as baseball and cricket (see Kruk, Regan, Beverley, \& Longridge, 1981; Regan, 1982, 1992). Given this usefulness of stereomotion, there may be clinical relevance in establishing thresholds for stereomotion, as has been done for stereop-

This research was supported by Natural Sciences and Engineering Research Council of Canada Grants A6313 to M.A.G. and A2600 to Z. W. Pylyshyn. L.A.S. is now in the Department of Psychology at Queen's University: W.S. is now in the Department of Psychology at Dalhousie University. Correspondence should be addressed to P. Servos, Department of Psychology, Wilfrid Laurier University, Waterłoo. ON, N2L 3C5 Canada (e-mail: pservos@machl.wlu.ca). sis (Scott \& Mash, 1974; Simons, 1981; Simons \& Reinecke, 1974; Walraven, 1975). Hong and Regan (1989), for example, reported that a substantial proportion of their observers had portions of the visual field which were stereomotion blind even though they had otherwise normal vision. The method that they used to establish this finding was optics based-a rather complicated methodology that is not commercially available. In recent years, the quality of computer graphics displays has increased to the point at which they might be comparable to more traditional optics-based displays. A portable display would make it possible to determine stereomotion thresholds in clinical or applied settings, given the availability of computers and the ease with which data can be collected and analyzed on them.

Like static stereopsis, stereomotion can be defined on the basis of either crossed or uncrossed disparities. There is some evidence that certain individuals are impaired in the processing of stereomotion stimuli defined by crossed disparities while showing relatively normal thresholds for stereomotion defined by uncrossed disparities. Individuals with the converse condition also exist (Hong \& Regan, 1989; Regan, Erkelens, \& Collewijn, 1986; Richard. \& Regan, 1973). Given these findings, we were interested in whether or not there might be differences between crossed and uncrossed stereomotion thresholds in a group of experimentally naive subjects.

The present study was conducted to ascertain whether or not a computer-graphics-based technique, involving liquid crystal (LC) glasses, can accurately assess stereomotion thresholds, and to discover any limitations (e.g., in the synchrony between the monitor display and the LC glasses) that such an approach might have relative to optics-based techniques. Given that such a technique might be a reasonable alternative to optics-based approaches, we also wanted to generate a normative data set of crossed and uncrossed stereomotion thresholds. 
To this end, two experiments were conducted. In one experiment, the stereomotion thresholds of 31 experimentally naive subjects were tested. In one condition, the stimuli were defined by crossed disparities, and in the other condition, they were defined by uncrossed disparities. In the other experiment, 4 experienced observers were tested in these two conditions as well as in conditions that assessed their sensitivities to translation and loom. The latter two conditions were used to assess whether or not the stereomotion thresholds of the experienced observers, as determined by this computer-based technique, might be influenced by non-disparity-based motion such as loom and translation.

\section{EXPERIMENT 1}

\section{Method}

Subjects. Thirty-one experimentally naive subjects (17 males, 14 females; mean age, 19.8 years; range, $18-25$ years) with normal or corrected-to-normal visual acuities participated for a research credit for an undergraduate course. All subjects had normal stereoacuity thresholds ( $40^{\prime \prime}$ of arc or better) as determined by the Randot Stereotest (Stereo Optical Co., Chicago).

Apparatus. All of the conditions were run on a Silicon Graphics Iris computer equipped with Stereoview LC eye-wear. The display screen was a 20-in. diagonal Silicon Graphics high-resolution monitor ( $85 \mathrm{dpi})$. Stereoscopic disparity was displayed by presenting images alternately to the left and right eyes at a rate of $120 \mathrm{~Hz} .^{1}$ The target was a red rectangle $1^{\circ}$ wide $\times 2^{\circ}$ high $\left(1.2 \mathrm{~cd} / \mathrm{m}^{2}\right)$ presented on a black background $\left(<0.01 \mathrm{~cd} / \mathrm{m}^{2}\right.$; as measured by a Minolta Chromameter Model CS- 100 through the LC glasses). A small veiling light was directed at the screen, raising the screen luminance to $45 \mathrm{~cd} / \mathrm{m}^{2}$. A red stimulus was chosen because red phosphor (the green and blue monitor guns were not used) decays faster than the other phosphors available on this sort of display. Two types of stereomotion stimuli were used: crossed and uncrossed. The crossed stimuli appeared to be oscillating back and forth in front of the screen; the uncrossed stimuli appeared to be oscillating back and forth behind the screen. To ensure that proper fusion occurred, and so that subjects could maintain fixation in the same depth plane as the screen, a centrally placed $8.0^{\circ}$ diameter circle was present throughout the testing session. If fusion was not achieved, this circle would break down into separate red and blue circles. A centrally placed fixation point (a plus sign subtending $0.33^{\circ} \times 0.33^{\circ}$ ) was present in between the trials.

The disparity-defined rectangle moved in square-wave steps at a $0.8-\mathrm{Hz}$ oscillation. The frequency and square-wave components of the stimuli were selected on the basis of previous research: The circumstances under which subjects are most sensitive to the presence of dynamic disparity information involve stimulus frequencies of approximately $0.8-1.0 \mathrm{~Hz}$ (see Regan \& Beverley, 1973a, 1973b, 1978, 1979; Richards \& Regan, 1973). In addition, subjects are more sensitive to square-wave oscillations, which present abruptly changing disparity information, than to sine-wave oscillations, which arrive at the maximum disparity level through a series of smaller changes in disparity. When retinal disparity was modulated sinusoidally, Regan and Beverley (1973a, 1973b) found a strong low-frequency attenuation below $1 \mathrm{~Hz}$, which was not present under square-wave modulation.

Procedure. Subjects wore the Stereoview glasses, and with proper fusion they perceived a single rectangle that appeared to be moving in depth. It should be emphasized that the physical size of the rectangle remained constant. Stimulus presentations lasted for $5 \mathrm{sec}$, after which time the subjects were required to respond.
Two counterbalanced testing blocks were used, one consisting of stimuli defined by crossed disparities, the other consisting of stimuli defined by uncrossed disparities. Data were collected with the dual randomly interleaved staircase technique, with one staircase starting at $24^{\prime}$ of arc and the other staircase starting at $0^{\prime}$ of arc, using 2-dB shifts (see Gescheider, 1985; Levitt, 1971). The subjects were required to indicate (using a mouse key) the presence of motion in the display, and the responses were recorded by the computer. For each of the two staircases, seven reversals were recorded, and the threshold estimate for each was determined by averaging the last six. The overall threshold was the average of the thresholds from the two staircases. Viewing distance was $114 \mathrm{~cm}$, and head movements were restricted by means of a chinrest equipped with two lateral head stops and a forehead stop. The psychophysical testing was preceded by the stereoacuity test. Each testing block lasted approximately $15 \mathrm{~min}$ and was performed in the dark.

\section{Results}

The mean stereomotion threshold for crossed disparities was $5.971^{\prime}$ of arc $(S E=0.515)$ and that for the uncrossed disparities was $6.002^{\prime}$ of $\operatorname{arc}(S E=0.454)$. Crossed and uncrossed stereomotion acuities were not significantly different $[t(30)=0.09, p>.9]$ but were positively correlated $(r=.77, p<.01)$.

\section{EXPERIMENT 2}

To produce the changes in disparity in Experiment 1, each eye was presented with a separate view of one of the two rectangles, which moved with respect to each other. In theory, some of the translational information in the rectangle yoked to, for example, the left eye might have been detected by the right eye (and vice versa), if the goggles were not perfectly synchronized with the displays, and it might have been this information and not changing disparity that was detected in the stereomotion tasks. If such lateral motion did leak into the stereomotion displays, it was also possible that this type of information could have made the stimulus appear to be looming in depth.

To assess whether or not 2-D motion information might have been affecting the stereomotion thresholds measured by the computer-graphics-based method, the translation and loom thresholds of 4 experienced observers were tested. Previous work with optics-based displays has shown that 2-D motion thresholds are lower than stereomotion thresholds (Hong \& Regan, 1989; Regan \& Beverley, 1973a, 1979). Thus, if the stereomotion thresholds in Experiment 2 were found to be as low as the 2-D motion thresholds, this would present the possibility that 2-D motion information might have been available in the stereomotion displays. If this were the case, one might expect practice effects in the stereomotion task; that is, subjects might gradually learn to make use of 2-D motion information when making their stereomotion judgments. To test for this possibility, stereomotion thresholds were measured over several testing blocks.

\section{Method}

Subjects. The 4 experimentally experienced subjects (all male; mean age, 27.0 years) were graduate student volunteers having nor- 


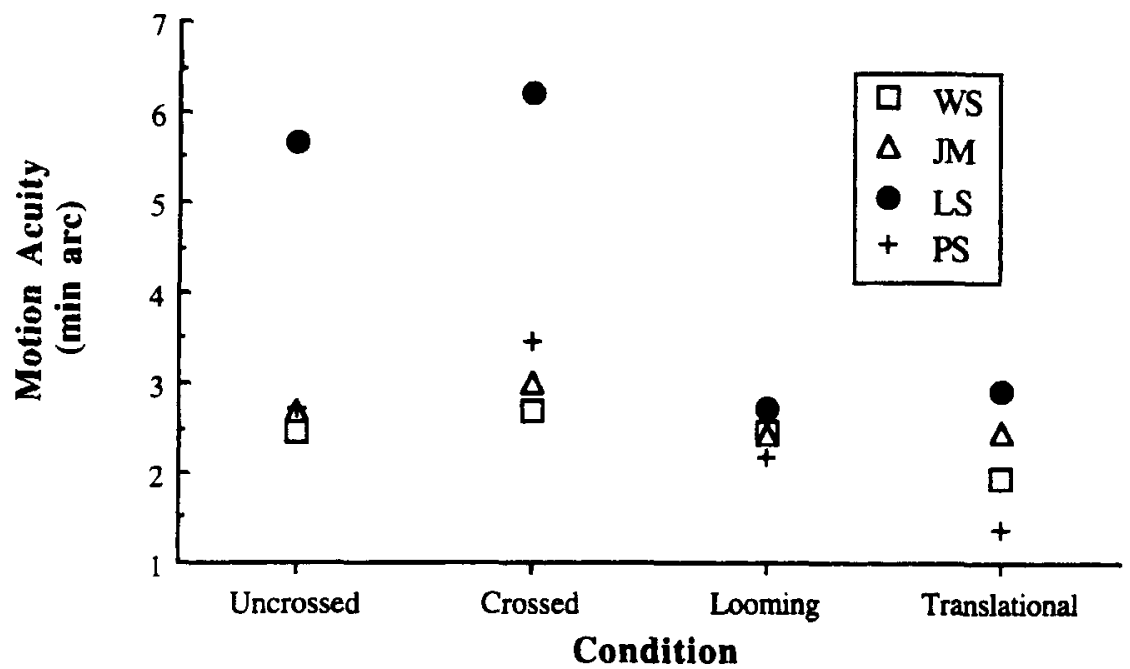

\begin{abstract}
Figure 1. Mean motion thresholds for the 4 experienced observers. In general, the translational motion thresholds (looming and translational) are lower than the stereomotion thresholds (crossed and uncrossed).
\end{abstract}

mal or corrected-to-normal visual acuity and stereoacuity thresholds better than $40^{\prime \prime}$ of arc.

Apparatus. The stimuli were presented on the Silicon Graphics Iris computer described in Experiment 1.

Procedure. In addition to the crossed and uncrossed stereomotion thresholds, translational motion and looming thresholds, under binocular viewing, were also collected in the 4 experienced subjects. In the translational motion condition, the 4 subjects were presented with a rectangle (similar in every way to the stereomotion stimulus, except that it was not defined by changing disparity) that moved from side to side within the frontoparallel plane. In the looming condition, a rectangle again was used, but this time it was made to increase or decrease in size, through manipulation of the length of its horizontal axis. In the latter two conditions, the squarewave oscillations were identical to those used for the stereomotion stimuli. The experienced subjects were given a total of six testing blocks for each of the four test conditions over 3 days, in counterbalanced order.

\section{Results}

The data for the experienced subjects were entered into a one-way repeated measures analysis of variance with four levels (crossed, uncrossed, loom, and translation). There was a significant main effect of condition $[F(3,9)=4.12, p<.05]$. Tukey's post hoc comparisons revealed that the crossed stereomotion thresholds were not significantly different from the uncrossed thresholds $(p>.05)$. The crossed stereomotion thresholds, however, were higher than the loom and translation thresholds [respectively, $t(9)=2.57, p<.05$, and $t(9)=3.06, p<$ .02 ; see Figure 1]. In addition, there was a strong trend suggesting that the uncrossed thresholds were also higher than the translation $[t(9)=2.21, p=.06]$ and loom $[t(9)=1.71, p=.12]$ thresholds, respectively. Translational motion thresholds did not significantly differ from the looming motion thresholds $(p>.05$; see Figure 1). The general pattern of stereomotion thresholds being higher than the thresholds for translation and loom was observed even when the data from the subject (L.S.) with the outlying stereomotion thresholds were removed from the analysis.

A further analysis was conducted to assess possible practice effects on the stereomotion thresholds reported here. The mean stereomotion threshold for the 4 experienced subjects was computed for each of the 12 testing blocks ( 6 testing blocks each for the crossed and uncrossed stimuli), and the data were entered into a oneway repeated measures analysis of variance. Early stereomotion thresholds could thus be compared with later thresholds. The thresholds for the testing blocks were not found to differ from each other $[F(11,33)=0.49, p>.8]$, suggesting that practice effects were not present.

\section{DISCUSSION}

Consistent with the work of Hong and Regan (1989), no differences in stereomotion thresholds were found between stimuli defined by crossed and uncrossed disparities. The actual stereomotion thresholds in the present study, however, appear to be somewhat lower than those reported by Hong and Regan in a comparable sample of inexperienced observers. The size of the stimuli used in the present study might account for this difference, in that they were slightly larger than those used by Hong and Regan (although they are comparable to some of the stereomotion stimuli used by Regan and his colleagues in other studies). In the Hong and Regan study, when stimuli were presented several degrees from fixation, there were slight increases in stereomotion sensitivitycomparable to the thresholds found in the present study. Presumably, our slightly larger stimuli would have subtended a region corresponding to the eccentricity used by Hong and Regan where maximal sensitivity occurred. The positioning of the stereomotion stimuli relative to 
the plane of fixation could have also produced differences in the thresholds observed in the present study and those reported by Hong and Regan. Their displays moved around a point $12^{\prime}$ of arc from the fixation plane in either crossed or uncrossed space. In accord with the convention adopted by Regan and Beverley (1973b), the stimuli in the present study always started at the plane of fixation and moved away from that point into crossed or uncrossed space. Consequently, the stereomotion thresholds of Experiment 1 in the present study are highly comparable to those reported by Regan and Beverley (1973a) for sine-wave oscillating stimuli, although higher than those reported in Regan and Beverley (1973b) for both square-wave and sine-wave oscillating stimuli. (It should be noted that most of the data in Regan and Beverley [1973a, 1973b] are based on only 1 subject. For the stimuli in the experiments closest to our own, this subject shows a range of thresholds from approximately $l^{\prime}$ of arc [Regan \& Beverley, 1973b] to 6' of arc [Regan \& Beverley, 1973a].)

A potential problem of measuring stereomotion thresholds with a computer-based display involving LC glasses is that any residual 2-D motion information might "leak" into the display (see Experiment 2), resulting in exaggerated stereomotion sensitivities. If this had been the case, one would have expected the stereomotion thresholds to have been as low as those for translational motion in the experienced subjects. Consistent with earlier, optics-based work, however, the translational and looming motion thresholds were found to be lower than the stereomotion thresholds (see Hong \& Regan, 1989; Regan \& Beverley, 1973a, 1979). This suggests that 2-D motion information did not contribute greatly to the stereomotion thresholds measured in the present study. Indeed, if residual 2-D motion information had been present in the stereomotion displays, some of the experienced observers should have been able to learn to use it over time. This was not the case, however, because no practice effects were observed. In addition, no "ghosting" was reported by the experimentally naive or the experienced subjects.

Regardless of the phenomenological experience of the subjects, it is possible that the synchrony between the monitor display and the LC glasses may not have been perfect, owing to phosphor persistence. The manufacturer of the system, Silicon Graphics, reports that the red phosphor used in their displays decays to $1 \%$ of background noise levels within $9 \mathrm{msec}$. Given that the screen was operating at $120 \mathrm{~Hz}(60 \mathrm{~Hz}$ for each eye), this would lead to a phosphor refresh every $8.3 \mathrm{msec}$, which could potentially lead to very slight ghosting in the other eye. As mentioned earlier, we did use a veiling light in the testing room, which produced light levels at the screen of $45 \mathrm{~cd} / \mathrm{m}^{2}$. It is likely that this would have minimized any ghosting. Moreover, LC shutters only allow $90 \%$ or so light transmission when they are "open." Thus, a 1-msec or shorter trace at around $1 \%$ intensity (i.e., around $0.12 \mathrm{~cd} / \mathrm{m}^{2}$ ) in the presence of a veiling light of $45 \mathrm{~cd} / \mathrm{m}^{2}$ and given the light transmission of the open shutters is a rather negligible stimulus. Finally, the unwanted $1 \%$ contrast difference, had it been present at all in the display, would not likely have been detectible because of contrast adaptation effects (Blakemore \& Campbell, 1969).

One of the main purposes of the present study was to determine the adequacy of a computer-graphics- and LCbased technique for assessing stereomotion thresholds. It appears that such displays can be used to assess stereomotion thresholds and may be a reasonable alternative to optics-based methods in clinical or applied settings.

\section{REFERENCES}

BEVERLEY, K. I., \& REGAN, D. (1973). Evidence for the existence of neural mechanisms selectively sensitive to the direction of movement in space. Journal of Physiology, 235, 17-29.

Beverley, K. I., \& Regan, D. (1975). The relation between discrimination and sensitivity in the perception of motion in depth. Journal of Physiology, 249, 387-398.

Blakemore, C., \& Campbell, F. W. (1969). On the existence of neurones in the human visual system selectively sensitive to the orientation and size of retinal images. Journal of Neurophysiology, 203, 237-260.

Gescheider, G. A. (1985). Psychophysics: Method, theory, and application (2nd ed.). Hillsdale, NJ: Erlbaum.

HoNG, X., \& REGAN, D. (1989). Visual field defects for unidirectional and oscillatory motion in depth. Vision Research, 29, 809-819.

Kruk, R., Regan, D., Beverley, K. I., \& Longridge, T. (1981). Correlations between visual test results and flying performance on the advanced stimulator for pilot training (ASPT) - I. Aviation, Space \& Environmental Medicine, 52, 455-460.

LEVITT, H. (1971). Transformed up-down methods in psychoacoustics Journal of the Acoustical Society of America, 49, 467-477.

REGAN, D. (1982). Visual information channeling in normal and disordered vision. Psychological Review, 89, 407-444.

REGAN, D. (1991). Depth from motion and motion in depth. In D. Regan (Ed.), Binocular vision (pp. 137-169). London: Macmillan.

REGAN, D. (1992). Visual judgements and misjudgements in cricket, and the art of flight. Perception, 21,91-115.

Regan, D., \& BeVERLEY, K. I. (1973a). The dissociation of sideways movements from movements in depth: Psychophysics. Vision Research, 13, 2403-2415.

Regan, D., \& Beverley, K. I. (1973b). Some dynamic features of depth perception. Vision Research, 13, 2369-2379.

REgaN, D., \& BEVERLEy, K. I. (1978). Looming detectors in the human visual pathway. Vision Research, 18, 415-421.

RegaN, D., \& Beverley, K. I. (1979). Binocular and monocular stimuli for motion-in-depth: Changing-disparity and changing-size feed the same motion-in-depth stage. Vision Research, 19, 1331-1342.

Regan, D., Beverley, K. I., \& Cynader, M. (1979). Stereoscopic subsystems for position in depth and for motion in depth. Proceedings of the Royal Society of London: Series B, 204, 485-501.

Regan, D., Erkelens, C. J., \& Collewijn, H. (1986). Visual field defects for vergence eye movements and for stereomotion perception. Investigative Ophthalmology \& Visual Science, 27, 806-819.

Regan, D., Frisby, J. P., Poggio, G. F., Schor, C. M., \& TYler, C. W. (1990). The perception of stereodepth and stereomotion: Cortical mechanisms. In L. Spillmann \& J. S. Werner (Eds.), Visual perception. The neurophysiological foundations (pp. 317-347). New York: Academic Press.

RICHARDS, W. (1972). Response functions for sine- and square-wave modulations of disparity. Journal of the Optical Society of America, 62, 907-911.

RICHARDS, W., \& REGAN, D. (1973). A stereo field map with implications for disparity processing. Investigative Ophthalmology \& Visual Science, 12, 904-909. 
ScotT, W. E., \& MASH, J. (1974). Stereoacuity in normal individuals. Annals of Ophthalmology, 6, 99-101.

SIMONs, K. (1981). Stereoacuity norms in young children. Archives of Ophthalmology, 99, 439-445.

SimONS, K., \& REINECKE, R. D. (1974). A reconsideration of amblyopia screening and stereopsis. American Journal of Ophthalmology, 78, 707-713.

Wal.raven, J. (1975). Amblyopia screening with random-dot stereograms. American Journal of Ophthalmology, 80, 893-900.

\section{NOTE}

1. The software for the presentation of the stereomotion stimuli is available by anonymous ftp at cogsi.uwo.ca (I.P. 129.100.6.10). Program names: crossed.c, uncrossed.c, and a support file in directory' pub/SGI.

(Manuscript received May 8, 1995; revision accepted for publication March 5, 1997.) 